\title{
Guide for Rational Use of Pediatric Antibiotics in Outpatient Clinic
}

\author{
Wang Shiguang \\ Department of medical function, Zhengzhou University of Industrial Technology, Zhengzhou China
}

Keywords: The clinic; Antibiotics; Children; The rational use of drugs

Abstract. In order to improve the level of using clinical drug, it is necessary to analysis the use of pediatric antibiotics in the clinic and make a guidance for rational use of drugs. Methods Selecting 90 cases of children as the research object, which have adverse reactions after antibiotic treatment in the outpatient clinic during 2012.4-2014.4. According to make a retrospective analysis of the children's clinical data, treatment, analysis and conclude the adverse reactions. Results The adverse reactions of pediatric antibiotics occur on the children between 2 and 6 years old $(56.67 \%)$. The incidence rate of the digestive system $(32.22 \%)$ and the respiratory system $(36.67 \%)$ is higher than others. The total number of unreasonable cases is $67(74.44 \%)$.Compare the adverse reactions incidence rate of different kinds of irrational usage of drugs, the difference is significant $\quad(\mathrm{P}<0.05)$

\section{Introduction}

With the medical technology develops increasingly, the new drugs become more and more. The antimicrobial agents, which is broad spectrum, low toxic and efficient is widely used in clinical treatment. In the using process of clinical medicine, the pediatric antibiotics can be used in different kinds of infection and reduce the mortality of infectious diseases ${ }^{[1]}$. The abuse of pediatric antibiotics is the most serious problem in the society, and the pediatric is in the first place. The common diseases of pediatric outpatient are respiratory tract infection, and the main is the upper respiratory infection ${ }^{[2]}$. If the pediatric antibiotics can be used properly, it can cure the respiratory tract infection and reduce the pain of the children, it can also reduce the incidence rate of adverse reactions. On the contrary, it will increase the state of an illness and the incidence rate of adverse reactions.

\section{Materials and methods}

General data.Selecting 90 cases of children as the research object, which have adverse reactions after antibiotic treatment in the outpatient clinic during 2012.4-2014.4. Among these, 49 cases of male, 41 female patients, they are between 0.3-14 years old, and the average age is (5.68 \pm 2.79$)$.

Methods. Using the retrospective analysis, investigate clinical preliminarily, looking up the medical records, make a detailed record of the gender, age, ward, bedID, admitting diagnosis, treatment condition, medication( Date, dosage, usage, purpose, etc.) Prevent and treat the adverse drug information and adverse reactions occurrence(time, cause, manifestation and treatment measures), regimen and adverse reactions. According to the survey, analysis the relevant factors with adverse reactions of pediatric antibiotics.

The evaluation criterion of adverse reactions. According to relevance evaluation of 6 levels which promulgated by the state food and drug administration adverse reactions monitoring center, the levels can be divided into certain ones, probably ones, possible ones, unrelated ones, for evaluating ones, unable to evaluate ones[3].

Statistical methods. Input the experimental data in professional statistical software SPSS14.0and completed the analysis of inspection, when $\mathrm{P}<0.05$, the statistical difference is significant. 


\section{Results}

Analysis of general data. 90 cases of adverse reactions in the children, men were slightly higher than women, but there is no obvious difference. Incidence rate of different ages is shown in the table 1.

Table 1 The gender and age distribution of children

\begin{tabular}{|c|c|c|c|c|c|}
\hline \multirow{2}{*}{$\begin{array}{l}\text { Age (years } \\
\text { old) } \\
0 \sim 30 \text { days }\end{array}$} & \multicolumn{2}{|c|}{ male percentage (\%) } & \multicolumn{2}{|c|}{ female percentage (\%) } & \multirow{2}{*}{$\begin{array}{l}\text { summation } \\
13 \text { (14.44) }\end{array}$} \\
\hline & 8 & 8.89 & 5 & 5.55 & \\
\hline 30days & 10 & 11.11 & 10 & 11.11 & $20(22.22)$ \\
\hline \multicolumn{6}{|l|}{2 years old } \\
\hline $\begin{array}{c}2 \sim 6 \text { years } \\
\text { old }\end{array}$ & 27 & 30.00 & 24 & 26.67 & $51(56.67)$ \\
\hline $\begin{array}{c}6 \sim 14 \text { years } \\
\text { old }\end{array}$ & 4 & 4.44 & 2 & 2.23 & $6(6.67)$ \\
\hline summation & 49 & 54.44 & 41 & 45.56 & $90(100.00)$ \\
\hline
\end{tabular}

Classification statistics of irrational drug usage .More than 90 cases of children with adverse reactions caused by irrational drug use, mainly displays in mutual antagonism between drugs, toxic additive, improper usage, improper dosage and so on. To compare adverse reactions incidence rate in different kinds of irrational drug use, when $\mathrm{P}<0.05$, the difference is significant, as shown in the table 2.

Table 2 The analysis of different dosing mode (n, \%)

\begin{tabular}{cccc}
\hline $\begin{array}{l}\text { Types of irrational } \\
\text { drug using }\end{array}$ & $\begin{array}{c}\text { cases of irrational drug } \\
\text { using }\end{array}$ & $\begin{array}{c}\text { The percentage of } \\
\text { selected children }\end{array}$ & $\begin{array}{c}\text { The percentage of irrational } \\
\text { drug using }\end{array}$ \\
\hline $\begin{array}{c}\text { mutual } \\
\text { antagonism } \\
\text { between drugs } \\
\text { toxic additive }\end{array}$ & 31 & 34.44 & 46.27 \\
$\begin{array}{c}\text { improper usage } \\
\text { improper dosage }\end{array}$ & 20 & 11 & 12.22 \\
summation & 5 & 22.22 & 29.85 \\
$\chi^{2}$ & 67 & 5.56 & 7.46 \\
p-value & - & 74.44 & 100.00 \\
& - & 20.24 & 27.92
\end{tabular}

Analysis of the main disease types caused by adverse reactions after antibiotic treatment. Analysis of the main disease types caused by adverse reactions after antibiotic treatment, mainly for children who are with upper respiratory tract infection, cold should use pediatric antibiotics, as shown in the table 3. 
Table 3 Diseases related with pediatric antibiotics

\begin{tabular}{ccc}
\hline Kinds of diseases & cases & percentage (\%) \\
\hline Upper respiratory tract infection & 5 & 5.56 \\
The common cold & 11 & 12.22 \\
Pneumonia & 13 & 14.44 \\
Acute sinusitis & 6 & 6.67 \\
Acute bronchial & 9 & 10.00 \\
Acute gastroenteritis & 16 & 17.78 \\
Acute diarrhea & 13 & 14.44 \\
Skin disease & 6 & 6.67 \\
Malnutrition & 11 & 12.22 \\
Summation & 90 & 100.00 \\
\hline
\end{tabular}

The severity and management of adverse reactions. Among of the 90 cases adverse reactions, the general degree is $76(84.44 \%)$,mainly displays in the respiratory system, mild reaction in the digestive system, the serious degree is $14(15.56 \%)$, mainly displays in upper respiratory tract infection, the common cold, pneumonia, acute sinusitis, acute bronchial, acute gastroenteritis, acute diarrhea, skin disease, malnutrition and so on.

Adverse reactions to relevance evaluation and the influence on the original diseases. Among of the 90 cases children, the number of certain cases is $56(62.22 \%)$, the number of probably cases is $23(25.56 \%)$, the number of possible cases is $11(12.22 \%)$.After symptomatic treatment, most children with adverse reactions become good, there is no obvious influence on the original disease.

\section{Discussion}

Penicillin, cephalosporin and macrocyclic lactones are used more than other antibiotics, among these, cephalosporin is used widely, because it has some characteristic, such as strong antibacterial effect, a broad antifungal spectrum, high clinical efficacy, resistance to penicillin enzyme, low toxicity and less anaphylaxis. The liver and kidney function, endocrine system, central nervous system are not fully developed, they are different from adults, and it is easy to cause drug allergy reaction and toxic effects ${ }^{[4-6]}$. So it is particularly important to properly use the pediatric antibiotics.

Through the study in this paper, 90 cases of adverse reactions in the children, men were slightly higher than women, but there is no obvious difference. $(\mathrm{P}>0.05)$.It proves that the occurrence of adverse reactions caused by outpatient pediatric antibiotics has no significant correlation with gender. Infants and young children, school-age children have higher outpatient rate, all ages are little changed. Adverse reaction is mainly concentrated in between 2 and 6 years old, they have the characteristics of urgency, quickness, and the condition changing complex. In the clinical application, facing the group, we should observe closely, have strict drug skin test, according to the results, then use the drugs ${ }^{[7-8]}$. Does not exclude the skin test results are negative, but the adverse reaction of children will still occur. This kind of situation is associated with drug treatment, allergic reaction is usually relatively quickly, if the rescue is not in time, it will kill the children. Even though the leaflet will emphasize the allergy banned, but most of the children couldn't determine drug allergy history; and even if there is no history of allergies, the children also cannot treat STH lightly. Therefore, if the children are used pediatric antibiotics whose skin test results are negative, they should be dealt with close observation to prevent allergic reactions leading to shock, and even kill the children ${ }^{[9-10]}$. From data analysis in table 2, the antibiotics utilization rate of our hospital outpatient pediatric is higher than others, and it has large relationship with the infection of the respiratory system diseases. The irrational drug usage mainly displays in mutual antagonism between drugs, toxic additive, improper usage, improper dosage and so on. Among these, the percent of mutual antagonism between drugs is the biggest 
$(46.27 \%)$, followed by improper usage $(29.85 \%)$ and toxic additive $(16.42 \%)$, finally for the use of improper dosage $(7.46 \%)$, when $\mathrm{P}<0.05$, the comparison is significantly different. The chemical injection drugs of antibiotics has complicated composition when different kinds of medicine are mixed together, it will cause a series of chemical changes. Drugs can essentially change, leading to reduce efficiency, precipitation, discoloration, toxic and other adverse reactions, and from the appearance, it is very difficult to find ${ }^{[11]}$. Therefore, the taboo of the pediatric antibiotics cooperated with other drugs needs textual research. Every medicine has its indications. Over the range, it will not only waste the resources, but also increase the incidence of adverse reactions. If the antibiotics is used not properly, it will waste the resources, at the same time, it will also damage to children's physical and mental health. So, in the process of clinical treatment, medical workers should improve their level of business. Upon on the specific conditions and the results of drug sensitivity of children, the medications should be reasonable and accurate, completely eradicate irrational drug usage. To provide children with a healthy, safe and effective solutions, ensure the drug efficacy and children's physical and mental health ${ }^{[12]}$.

To sum up, in the use of pediatric antibiotics exceed the scope of indications(antibiotic also be used in the common cold, malnutrition and so on ), drug usage is irrational. Because of the management of drug usage is weak, clinical application is unreasonable. To ensure the drug usage is reasonable, ensuring the safety, effectiveness and economy of drug usage of children has become the problem of the major medical institutions, especially the abuse of antibiotics has led to countries to attach great importance to the agency. In the moment, Ministry of Health has increased the management strength for antibiotics and promulgated the constraint for laws and regulations, it has made some progress, but it still needs to strengthen supervision, to ensure the clinical rational drug usage. In the meantime, the medical department should increase the publicity of antibiotic drug related knowledge, ensure that medical staff and families of children can correct use pediatric antibiotics, and put an end to drug abuse fundamentally.

\section{Conclusion}

Because of the adverse reactions incidence rate of pediatric antibiotics is very high, we should pay attention to it. Ensure the rational use of clinical drugs to increase the safety of the using drugs.

\section{References}

[1]Hu Daohua, Hu Yongquan, Yu Meiling etc. The 1st affiliated hospital of Bengbu medical college. Outpatient pharmacy prescription comment from January to June in 2012. [J]. Drug evaluation research, 2014,37(1):70-73.

[2]Diaz,L.,Ciurea, A.M..Cutaneous and systemic adverse reactions to antibiotics[J].Dermatologic therapy,2012,25(1):12-22.

[3]Jia Tianhua, Li Zhiping. The survey of 365 cases of pediatric patients using antibiotic adverse reaction. [J]. Chinese drugs of economics. 2013,16(4):326-327.

[4]Huang Xiaodan. 4 cases of adverse reaction caused by Cefonicid sodium. [J]. The Chinese hospital pharmacy magazine, 2013,33(10):835-836.

[5]Wang Hongyan. The reasonable application and the analysis of measures of pediatric antibiotics.[J].Journal of clinical rational drug use. 2013,6(12):35-35.

[6]Yuan Shenghong. The survey of adverse reaction caused by aminoglycoside antibiotics. [J]. Chinese practical medicine. 2015,2(3):170-171.

[7] Picard,M.,Paradis,L.,Nguyen,M. et al.Outpatient penicillin use after negative skin testing and drug challenge in a pediatric population[J].Allergy and asthma proceedings,2012,33(2):160-164. 
[8]Huang Yufei, Cephalosporin antibiotics in the clinical application of pediatric upper respiratory tract infection. [J]. Yanbian medical. 2014,9(36):210-211.

[9]Zhao Libo. The research of pediatric drug adverse reaction. [J]. Clinical rational drug use magazine. 2013,6(12):21-22.

[10]Liu Dong. The analysis of application effects and adverse reactions of pediatric antibiotics. [J]. China's health standard management. 2015,5(2):61-62.

[11]Wang Xiaojun. The observation of adverse reactions of pediatric antibiotic used on children. Massage and rehabilitation medicine. 2014,20(9):116-116,117.

[12]Rong Haili. The value analysis of reasonable use of pediatric antibiotic. Chinese medicine guide. 2012,10(20):567-568. 